\title{
Reduction of Amplitude Ratio Dependence on Drive Power Variations in MEMS Mode Localized Sensors
}

\author{
Milind Pandit, Chun Zhao, Guillermo Sobreviela, Arif Mustafazade, Ashwin A Seshia. \\ Nanoscience Centre $1 \mathrm{JJ}$ Thomson Avenue \\ University of Cambridge \\ Cambridge, UK \\ E:mnp26@cam.ac.uk
}

\begin{abstract}
For the first time, this paper highlights the dependence of amplitude ratio on the drive power in mode localized sensors. The simulation and experimental results show that the amplitude ratio measurements are most insensitive to drive power variations in the veering zone (where the two resonators are matched in stiffness and mass). This observation is a potential pathway to high resolution long term measurements in mode localized sensing.
\end{abstract}

Keywords-MEMS; Mode localization; Resonators; Veering; Resolution

\section{INTRODUCTION}

Mode Localization in weakly coupled resonators (WCRs) has been recently developed as an ultra-sensitive technology for gravitation, acceleration, charge and mass measurements. Unlike conventional frequency shift sensing in single resonator devices, WCRs measure amplitude ratio or Eigenstate shifts that can achieve orders of magnitude higher sensitivity [1-3]. Additionally, they employ common mode rejection properties that provide immunity to first-order response from ambient temperature and pressure variations [4-5].

To date, mode localized sensors have only been characterized in the linear domain at low vibration amplitudes. Nonlinear models of mode localization are complex and their solutions are difficult to obtain analytically. Furthermore, high vibration amplitudes introduce duffing effects in the system that lead to amplitude-frequency (a-f) dependency where the closed loop stability of the oscillator can be affected negatively [6]. However, there are several advantages of operating the sensor in high vibration amplitudes. In a system dominated by electrical noise of the readout electronics, high vibration amplitudes allow for a greater output signal thus resulting in high signal to noise ratio (SNR). This potentially could lead to higher resolution amplitude measurements for amplitude ratio sensing [3].

In this paper, we show through experiment and simulation for the first time, that the amplitude ratio output has a dependency on the drive power in the resonator's nonlinear regime. We then demonstrate that by operating the mode localized sensor in the veering zone, the amplitude ratio becomes most immune to drive power variations even at high vibration amplitudes (beyond bifurcation). This results in high
SNR with minimal impact on the ability of the system to perform as a sensor. This study represents a first step towards optimizing the resolution of mode localized sensors in a close loop configuration [7].

\section{THEORY}

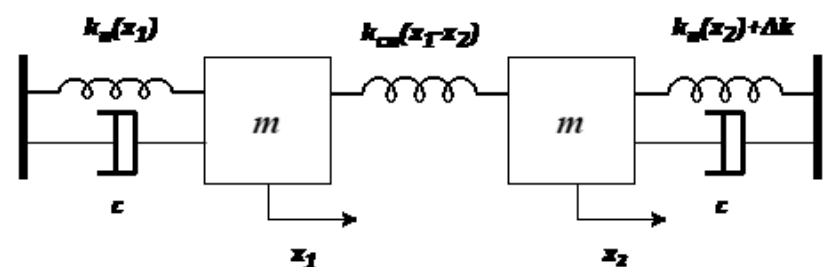

Fig. 1. Discrete nonlinear spring-mass system

The WCRs can be modelled using a discrete spring-mass system as outlined in Fig. 1. All of the springs including the coupler are assumed to be nonlinear up to the third order. The damping constant is assumed to be linear and no damping is assumed in the coupler as it is electrically coupled. Such a system has two vibration modes - the in-phase mode and the anti-phase mode. In the linear operating range, it has been shown that when the two resonators are completely matched in stiffness and mass, the anti-phase mode is separated from the in-phase mode by a factor proportional to the coupling stiffness. Stiffness perturbations in ether of the springs, leads to a veering of the Eigenvalue from this point and so the matched scenario is called the veering point. The amplitudes of vibration of the two resonators for a particular mode are also matched at this point and the amplitude ratios of the two modes are equal to 1 . Any perturbations in the stiffness of either resonator change the amplitudes of the two resonators and leads to a shift in the amplitude ratio of the system. This leads to a profile of the amplitude ratio and the frequency against the stiffness perturbations that have been very well studied in the linear operating regime [1-5].

The profile of the amplitude ratio shift has not been characterized yet so far in the nonlinear operating regime. In this work, the profile is studied closely through simulations in Simulink $^{\circledR}$ and experiments on Silicon on Insulator (SOI) weakly coupled resonators. 


\section{SimUlation}

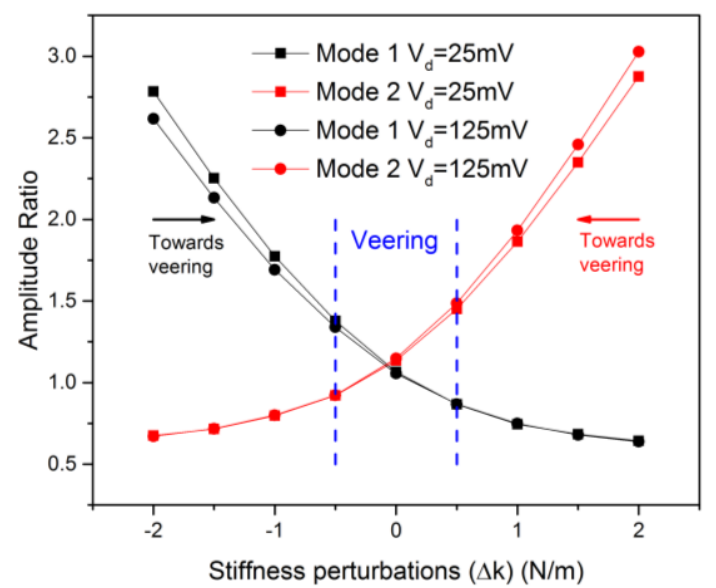

Fig. 2. Simulation results of amplitude ratio at two drive voltages across a range of stiffness perturbations

The nonlinear springs, mass and damper are used to create a simple nonlinear model in Simulink ${ }^{\circledR}$ based on transfer function analysis. The drive amplitude $\left(\mathrm{V}_{\mathrm{d}}\right)$ is changed from $20 \mathrm{mV}$ to $125 \mathrm{mV}$ while maintaining a coupling voltage of $15 \mathrm{~V}$. The response is shown in Fig. 2.

The simulation for the first time shows that the amplitude ratio is a function of the $\mathrm{AC}$ drive voltage. This introduces a potential issue where amplitude noise in drive voltage can be converted in the form of amplitude ratio variation, hence reducing the resolution. Based on this observation, we are able to extend the expression of amplitude ratio noise as follows [3]:

$$
\left\langle\frac{X_{1}}{X_{2}}\right\rangle^{2}=\left|\frac{X_{1}}{X_{2}}\right|^{2}\left[\left(\frac{X_{n, 1}}{X_{1}}\right)^{2}+\left(\frac{X_{n, 2}}{X_{2}}\right)^{2}\right]+f\left(\left\langle V_{n, d}\right\rangle^{2}\right)
$$

In (1), $\left\langle X_{1} / X_{2}\right\rangle$ is the noise of amplitude ratio, $X_{n, 1}$ and $X_{n, 2}$ are the amplitude noise of resonator 1 and 2 respectively. The additional term $f\left(\left\langle V_{n, d}\right\rangle\right)$ represents the conversion between the drive power variations to amplitude ratio noise. By operating the resonators in nonlinear regime, the terms $\left|X_{n, 1} / X_{1}\right|$ and $\left|X_{n, 2} / X_{2}\right|$ can be reduced. However, to achieve higher resolution measurements, a reduction in the additional term of $f\left(\left\langle V_{n, d}\right\rangle\right)$ is required.

It can be noticed that the amplitude ratio dependence on drive amplitude is less prominent near the veering area $(|\Delta k| \leq$ $0.5)$. Thus, operating the sensor in the veering region allows for reduction in $f\left(\left\langle V_{n, d}\right\rangle\right)$ and potentially increase the resolution of the sensor. This trend is shown in both the modes and is not restricted to any features found in a particular mode. To verify this effect seen in the simulation results, further experiments are performed.

\section{EXPERIMENTAL SETUP}

The optical micrograph of the device used for the experiment is shown in Fig. 3. Two Double Ended Tuning Fork (DETF) MEMS resonators are electrically coupled using DC voltage between their capacitive plates. Resonator 1 is

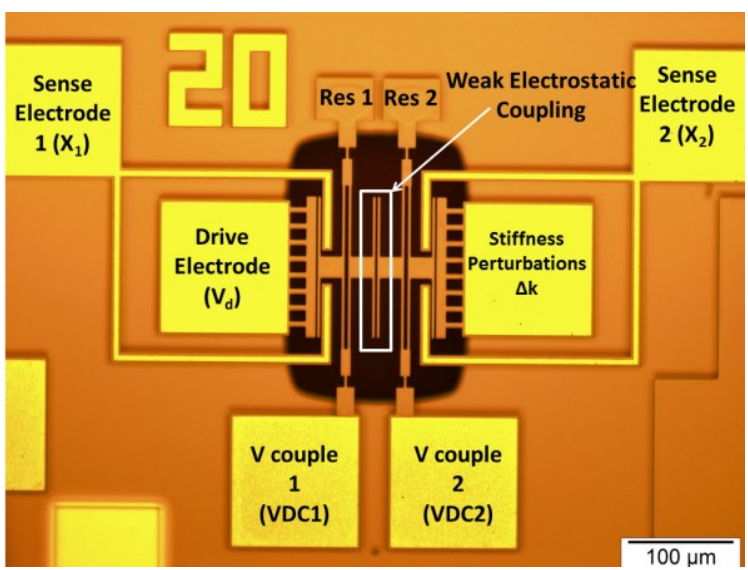

Fig. 3. Optical micrograph of the device

driven capacitively from the drive electrode with a combination of an AC voltage and a DC polarising voltage. The two sense electrodes sense the mechanical vibration of the two resonators $\left(X_{1}\right)$ and $\left(X_{2}\right)$. Negative stiffness perturbations are added on resonator 2 by sweeping the dc bias on the perturbation electrode. The sense current from $\left(X_{1}\right)$ is fed through Zurich Instruments PLL (MFLI) to close the loop on resonator 1 while resonator 2 is driven through the coupler. The nominal resonant frequency of the device is $250 \mathrm{kHz}$.

\section{EXPERIMENTAL RESULTS}

The resonator system was operated in vacuum to maximize the quality factor. The coupling voltage is set to $8 \mathrm{~V}$. Stiffness perturbations are applied on resonator 2 while measuring the amplitude and frequency data of the resonators. The AC drive voltage is changed from $20 \mathrm{mV}$ to $50 \mathrm{mV}$ and $70 \mathrm{mV}$ to reflect the linear drive amplitude $\left(\Delta f_{\text {peak }} / f_{3 d b} \sim 0\right)$, critical drive amplitude $\left(\Delta f_{\text {peak }} / f_{3 d b} \sim 0.5\right)$ and nonlinear drive amplitude $\left(\Delta f_{\text {peak }} / f_{3 d b} \sim 1\right)$ of the resonator system respectively. These results are plotted in Fig. 4 and the percentage variations are

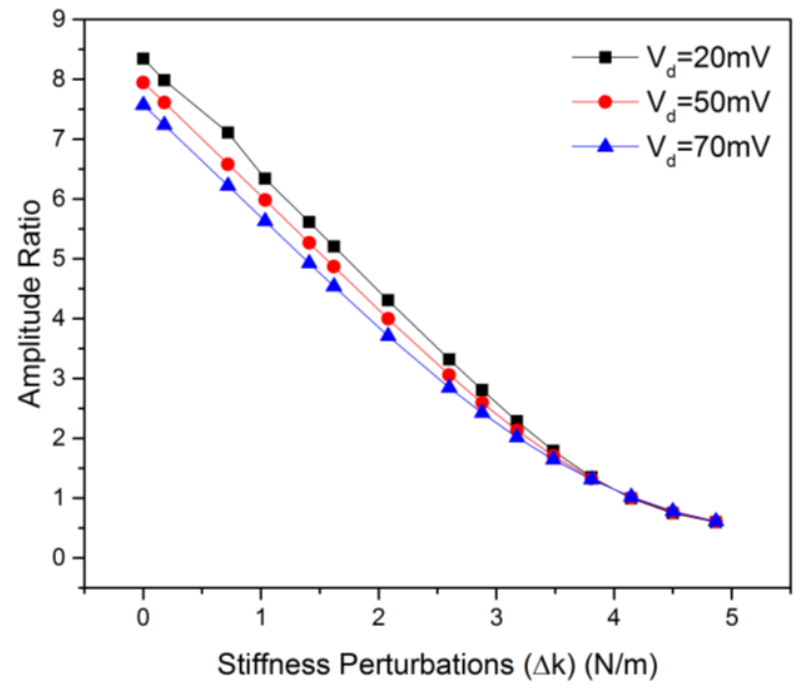

Fig. 4. Comparison of Amplitude Ratio at different drive voltages across a range of stiffness perturbations 
delineated in Tab. I for three points at different regions in the spectrum.

TABLE I. AMPLITUDE RATIO VARIATIONS ACROSS VARIOUS DRIVE VOLTAGES.

\begin{tabular}{|c|c|c|c|}
\hline & $\boldsymbol{V}_{\boldsymbol{d}}=\mathbf{2 0} \boldsymbol{m} \boldsymbol{V}$ & $\boldsymbol{V}_{\boldsymbol{d}}=\mathbf{5 0 \boldsymbol { m } \boldsymbol { V }}$ & $\boldsymbol{V}_{\boldsymbol{d}}=\mathbf{7 0 \boldsymbol { m }} \boldsymbol{V}$ \\
\hline Veering & 0.994775 & 1.009487 & 1.021169 \\
$(\mathbf{A R} \sim \mathbf{1})$ & $(-)$ & $(1.48 \%)$ & $(2.65 \%)$ \\
\hline $\begin{array}{c}\text { Near Veering } \\
(\mathbf{1 . 5}<\mathbf{A R}<\mathbf{5})\end{array}$ & 3.316283 & 3.060178 & 2.844566 \\
$(-)$ & $(7.72 \%)$ & $(14.23 \%)$ \\
\hline Far from & 7.108513 & 6.581116 & 6.220521 \\
veering & $(-)$ & $(7.42 \%)$ & $(12.49 \%)$ \\
$(\mathbf{A R}>\mathbf{5})$ & &
\end{tabular}

The initial condition of the resonator amplitude ratio without any perturbations is $>>1$. This is mainly due to the manufacturing tolerances and the electrostatic spring softening effect on the capacitive plates in the system. As the stiffness perturbations are added, the amplitude ratio decreases close to 1 and then continues to decrease upon further perturbations. As this process is repeated for the different drive voltages, the amplitude ratio deviates from the linear case at high amplitude ratios $(\sim 15 \%)$ while they deviate very little close to veering zone $(<3 \%)$. Our results show that the amplitude ratio is most immune to drive power in the vicinity of the veering zone (amplitude ratio $\sim 1$ ) as compared to away from the veering zone (amplitude ratio $>>1$ ). Furthermore, operating the sensor away from the veering point (amplitude ratios $>>1$ ) leads to one of the resonator amplitudes being far lower than the other. Since the system is dominated by electrical noise, the resolution of the readout will be limited by the resonator with lower amplitude. However, operating the sensor around the veering point, allows for potentially higher output resolution since both the resonators will have similarly high output voltages.

\section{DISCUSSION}

When the WCRs are driven only from one end, they work on the master-slave principle. So, the amplitude of the master resonator controls the nonlinear behaviour of the system. Thus, when the system is unmatched and is biased away from veering, one of the resonators has a much higher amplitude of vibration that the other. However, if the system is now driven into nonlinearity, the unstable frequency response is seen in both the resonator responses (even though the slave resonator is not vibrating above its critical amplitude). This results in a disproportional increase in the amplitude of vibration of the slave resonator as compared to the master resonator when the drive voltage is changed and thus leads to variation in the amplitude ratio. However, in the case that the two resonators are matched, the amplitudes of vibrations of the two resonators are similar. Now when the drive voltage is increased, it leads to proportional change in the amplitudes of both resonators and thus reduces the sensitivity of the amplitude ratio to drive voltages.

\section{CONCLUSION}

This work highlights the dependence of amplitude ratio in mode localized sensors to drive power variations. The sensor is less sensitive to variations in the drive power near the veering zone where the two resonators are closely matched in stiffness and mass. Operating the sensor in this region reduces the conversion of drive power noise to output noise. Potentially it paves the way for high resolution measurements due to the increased SNR without compromising the working of the sensor.

\section{ACKNOWLEDGMENT}

This work is supported by funding from Innovate UK and Natural Environment Research Council.

\section{REFERENCES}

[1] P. Thiruvenkatanathan, J. Yan and A. A. Seshia, "Enhansing Parametric Sensitivity in Electrically Coupled MEMS Resonators," J. Microelectromechanical Syst. , vol. 18, no. 5, pp. 1077-1086, October 2009.

[2] C. Zhao, G. S. Wood, J. Xie, H. Chang, S. H. Pu and M. Kraft, "A force sensor based on three weakly coupled resonators with ultrahigh sensitivity," in Sens. Actuators Phys., vol. 232, pp. 151-162, Aug. 2015.

[3] H. Zhang, B. Li, W. Yuan, M. Kraft and H. CHang, "An Acceleration Sensing Method Based on the Mode Localization of Weakly Coupled Resonators," J. Microelectromechanical Syst. , vol. 25, no. 2, pp. 286296, April 2016.

[4] P. Thiruvenkatanathan, J. Yan and A. A. Seshia, "Differential amplification of structural perturbations in weakly coupled MEMS resonators," IEEE Trans. Ultrason. Ferroelectr. Freq. Control, vol. 57, no. 3, pp. 690-697, March 2010.

[5] H. Zhang, J. Zhong, W. Yuan, J. Yang and H. Chang, "Ambient pressure drift rejection of mode localized resonato sensors," in 2017 IEEE $30^{\text {th }}$ International Conference on Micro Electro Mechanical Systems (MEMS), 2017, pp. 1095-1098.

[6] G. Sobreviela, G. Vidal-Álvarez, M. Riverol, A. Uranga, F. Torres and N. Barniol, "Suppression of the A-f mediated noise at the top bifurcation point in a MEMS resonator with both Hardening and softening hysteretic cycles," Sens. Actuators Phys., vol. 256, pp. 59-65, Aprl. 2017.

[7] C. Zhao, M. Pandit, B. Sun, G. Sobreviela, X. Zou, and A. Seshia, "A Closed-Loop Readout Configuration for Mode-Localized Resonant MEMS Sensors," J. Microelectromechanical Syst., vol. 26, no. 3, pp. 501-503, Jun. 2017. 